consultation with a family member participating and 52.9\% had included an external health professional. Wellbeing staff had also successfully provided multiple group support sessions via video for both patients and carers. The respondents thought video consultations were efficient and convenient for hospice professionals (80.6\%) and patients (67.7\%). As a consequence of the rapid shift to video consultations, our results highlighted that $78 \%$ of respondents had received no formal training, and in addition, 39\% reported some technical difficulties. Overall $80.7 \%$ wanted to offer video consultations as an option in the future.

Conclusion Hospice professionals have quickly adapted to video consultations and are keen to continue to offer this service in the future but need appropriate training and reliable videoconferencing technology in order to do this effectively. Videoconferencing can be used in creative ways to expand access for patients and family caregivers to a range of palliative care services and enhance multi-professional teamworking.

\section{A SERVICE EVALUATION OF VIDEO CONSULTATIONS IN A UK HOSPICE DURING THE COVID-19 PANDEMIC: LEARNING FROM PATIENTS' AND CARERS' FEEDBACK}

Shannon Milne, Jennifer Palfrey, Jane Berg, Jennifer Todd. Princess Alice Hospice, Esher

\subsection{6/spcare-2021-PCC.132}

Introduction During the Covid-19 pandemic, our hospice services had to rapidly move to consulting using video-conferencing technology. A service evaluation captured our patients' experiences of video consultations provided by a range of hospice professionals.

Methods An online or postal survey was sent to a convenience sample of patients, who had participated in video consultations between March and June 2020, focussing on their experience and overall satisfaction with the service.

Results We received 26 responses from patients (23) and family members/carers (3), 12 of whom were over the age of 70. Patients had consulted with our doctors (2), nurses (14), physiotherapists (5), occupational therapist (1), social worker (1), complementary therapist (4) and Wellbeing team (17). 25 respondents stated that they used the internet daily, 22 had access to a smartphone, and 23 used videoconferencing for reasons other than healthcare. However, 22 had never used this technology for healthcare prior to the pandemic. $32 \%$ included a family member in their consultation and this was viewed as beneficial. $88.4 \%$ experienced being able to talk in the same way as if they were face-to-face. All respondents reported overall satisfaction with consulting via video, although $41.7 \%$ still stated a preference for face-to-face consultations where possible. $91.7 \%$ said they would be happy to use video to consult with hospice professionals in the future, and $87.5 \%$ stated that they would recommend this service video to others.

Conclusions Patients and carers reported video consultations to be an acceptable way to receive support from a range of hospice professionals. The majority had access to, and familiarity with their own technology. Video consultations should be offered as an alternative to face-to-face consultations with the potential to improve access for more patients and carers to a range of hospice services in the future.

\section{TIME FOR A CHANGE? TRANSFORMING THE INVESTIGATION AND MANAGEMENT OF ANAEMIA IN THE PALLIATIVE CARE POPULATION}

Sophie Jones, Claire Stark Toller. Mountbatten Hampshire

\subsection{6/spcare-2021-PCC.133}

Background Severe anaemia is common in the hospice setting. Causes include anaemia of chronic disease (ACD) and associated functional iron deficiency (FID), with or without absolute iron deficiency anaemia (IDA). National guidance advises anaemia is investigated and treated to reduce need for blood transfusion. ${ }^{1}$ However, this is infrequently done. ${ }^{2}$ Blood transfusion is used to treat symptomatic anaemia but there is limited evidence on effectiveness and duration of response., ${ }^{3,4}$ Harm of transfusion is well documented; even a single unit risks transfusion associated circulatory overload (TACO). ${ }^{5}$ Consequently, guidelines were introduced to an inpatient palliative care unit to ensure anaemia was investigated, IV iron prescribed appropriately, and a restrictive transfusion strategy based on weight adopted.

Method Retrospective data collection from patients admitted for blood transfusion over one year: investigations (iron studies, haematinics, CRP); IV iron prescriptions. Risk factors for TACO were collated.

Results 37 units were administered to 15 patients. 58\% had one unit; the remaining had two. Mean pre-transfusion haemoglobin $67 \mathrm{~g} / \mathrm{L}(44 \mathrm{~g} / \mathrm{L}-84 \mathrm{~g} / \mathrm{L})$. Mean post-transfusion haemoglobin $78 \mathrm{~g} / \mathrm{L}(43 \mathrm{~g} / \mathrm{L}-125 \mathrm{~g} / \mathrm{L})$; checked in $54 \%$ patients. $50 \%$ patients had $<5 \mathrm{~g} / \mathrm{L}$ improvement in haemoglobin. Iron studies and haematinics were requested in $73 \%$ patients; at least one requested in 92\%. IDA was identified in one patient, FID identified in four, and a combination IDA/FID in two. Five had no deficiency. Of the seven with IDA or FID, five received blood and iron infusions. 100\% patients had one risk factor for TACO; $47 \%$ had two and $27 \%$ had three. $50 \%$ had a weight recorded within 2 months of transfusion.

Conclusion Guideline introduction increased investigation into causes of anaemia. Identification of FID/IDA enabled treatment with IV iron and blood transfusion. Prior to guideline introduction, IV iron had not been prescribed. High risk of TACO mandates a cautious approach to blood transfusion and supports a restrictive transfusion strategy.

\section{REFERENCES}

1. NICE (2015) Blood transfusion. National Guideline NG24. www.nice.org.uk

2. Neoh K, et al. (2019) National comparative audit of red blood cell transfusion practice in hospices: Recommendations for palliative care practice. Palliative Medicine.33:1021â€' '08.

3. Uceda Torres ME, et al. (2014) Transfusion in palliative cancer patients: A review of the literature. Journal of Palliative Medicine.17: 88â€ '104.

4. Chin-Yee $\mathrm{N}$, et al. (2018) Red blood cell transfusion in adult palliative care: a systematic review. Transfusion. 58: 233â€ '241.

5. SHOT. Annual SHOT Report 2019. https://www.shotuk.org/resources/currentresources (accessed 20th December 2020).Save

\section{PALLIATIVE CARE PROVISION ON THE INTENSIVE CARE UNIT: A SERVICE EVALUATION OF IDENTIFICATION OF NEED, METHOD OF DELIVERY, AND QUALITY OF CARE}

${ }^{1}$ Stephanie A Hill, ${ }^{1}$ Abdul Dawood, ${ }^{1}$ Elaine Boland, ${ }^{1}$ Hannah Leahy, ${ }^{2}$ Fliss EM Murtagh. ${ }^{1}$ Hull University Teaching Hospitals NHS Trust; ${ }^{2}$ Wolfson Palliative Care Research Centre, Hull York Medical School, University of Hull

10.1136/spcare-2021-PCC.134 